\title{
¿Ocaso de la idea de sociedad?
}

\section{Francois Dubet*}

Para comenzar, dos observaciones. La primera tiene que ver con el carácter de la idea misma de sociedad, la que aparece como una filosofía social, una manera de responder al problema de la modernidad. La idea de sociedad se impone en el pensamiento social de fines del último siglo, cuando el orden social ya no se percibe como natural o divino por una parte, y cuando el cambio social aparece tan rápido y tan destructor que las sociedades se ordenan en una sucesión de formas históricas, por la otra. La idea de sociedad se impone, entonces, como una definición conjunta de la totalidad y del cambio, cuando las respuestas del siglo XVIII, en términos de mercado y de contrato social, parecen insuficientes (Nisbet 1984). La idea de sociedad es indisociable de una filosofía social de la modernidad.

La segunda observación concierne al ámbito de las teorías sociológicas contemporáneas en el cual la idea de sociedad está ausente o es débil. Las teorías dominantes son de inspiración posmodernas, neoliberales o interaccionistas. Todas estas teorías ahorran ampliamente la idea de sociedad. ¿Podemos por eso abandonar la idea de sociedad, podemos construir sociologías excluyendo la idea de sociedad?. No creo que eso sea posible, incluso si la idea de sociedad debe ser hoy día profundamente transformada porque ya no es identificable a un orden "natural".

\section{LA IDEA DE SOCIEDAD COMO NARRACIÓN DE LA MODERNIDAD}

La idea de sociedad está en el centro de la tradición sociológica "clásica" en la cual el orden social y la modernidad están fuertemente integrados. Que los "padres fundadores" sean optimistas o pesimistas, progresistas o conservadores, no

CADIS, EHESS. UNIVERSITE DE BORDEAUX $\|$.

Este articulo lue elaborado durante la estadia del autor como Profesor Visitante del Departamento de Sociología de la Universidad de Chile, en noviembre de 1995. Traducido dei francés por Jaime Aymerich con la colaboración de M.A.Garretón. 


\section{REVISTA DE SOCIOLOGIA}

quita que todos ellos se apoyen en una representación de la sociedad que implica algunos elementos estables. Distinguiremos cuatro:

\section{Las dimensiones de la sociedad}

\section{a) La sociedad es moderna}

La idea de sociedad identifica la sociedad a la modernidad. Todo el pensamiento clásico reposa sobre una pareja de oposiciones, de las cuales Tonnies trazó la matriz esencial: la oposición entre la comunidad y la sociedad. Bajo formas diversas ésta se encuentra en todos los grandes pensamientos sociológicos. Como las dos formas de solidaridad, mecánica y orgánica, de Durkheim. Como principio organizador de los tipos de acción en Max Weber y la teoría de la racionalización. También las "patterns variables" en Parsons. En una cierta medida incluso como los "estadios" del desarrollo en Marx. Para todos, la idea de sociedad está asociada al reino de la racionalidad y de la razón contra lo sagrado y la costumbre. Para todos, la sociedad es compleja, racional, individualizante y fría. Además, numerosos sociólogos denuncian los riesgos de la alieneación vinculados a la sociedad, la anomia, el desencantamiento, la soledad de las masas. En una importante medida, la idea de sociedad fué también portadora de una crítica social de la modernidad, aunque mantiene la idea que la sociedad está apliamente fundada en una representación evolucionista del cambio y en la creencia en la necesidad de la modernización de la cual las sociedades industrializadas han trazado la vía. El penasamiento desarrollista de los años cincuenta en América Latina es ejemplar a este respecto.

\section{b) La sociedad es un sistema.}

Para una corriente esencial de la sociología clásica (Durkheim, Parsons) la sociedad es un sistema, una totalidad organizada de funciones que garantizan la integración del conjunto. La complejidad de la modernidad es reducida por la integración funcional y, en gran medida, la idea de sociedad es funcionalista, tanto si se trata de un organicismo simple o de un funcionalismo elaborado (Davis,1968 Merton 1965). Explicar las conductas sociales es explicar para qué siven, en qué contribuyen a la integración del conjunto, ya se trate de la división del trabajo o de las diversas instituciones que tienen por función integrar los individuos en el conjunto social; la familia, la escuela, la religión. La integración de los individuos y la integración del sistema aparecen como las dos caras de un mismo conjunto.

\section{c) La sociedad es un Estado Nacional.}

La idea de sociedad es puramente abstracta y detrás de ella está, "en realidad", la identificación de la sociedad y el Estado nacional moderno. Dicho de otra ma- 
conjunto, son las vertientes subjetiva y objetiva de la misma realidad. Los actores son producidos por la sociedad y producen la sociedad actuando según el programa de su socialización. Ninguna trascendencia es entonces necesaria para explicar el orden social; se puede, por lo tanto "explicar lo social por lo social". Durkheim y Parsons son los que más han desarrollado esta idea mostrando que había un principio de continuidad entre los valores y la personalidad. En este sentido la idea de que la sociedad es una realización práctica de los actores está lejos de ser nueva.

Esta representación lleva a colocar en el centro del análisis dos nociones; socialización e institución.

La socialización es una suerie de equivalente universal de la sociología porque permite explicar el paso del sistema a los actores. Explicar las conductas, es decir, cómo fueron socializadas, es mostrar cuáles aprendizajes y qué códigos culturales están detrás de las conductas a priori espontáneas: los gustos, la elección del cónyuge, el voto.

Hoy día es ya banal explicar las conductas y opciones haciendo corresponder posiciones sociales y actitudes por la vía indirecta de la socialización. Es también el esquema suyacente del análisis de correspondencia.

La noción de institución está ligada a la de socialización y consiste en definir los aparatos y las organizaciones por su capacidad de asegurar a la vez la integración del sistema y la de los actores. La socialización es percibida entonces como un efecto institucional. Conviene subrayar que esta representación no es ni cínica ni puramente sociológica. El actor no es un clon, sino también un sujeto ya que la sociología clásica afirma la continuidad entre la socialización y construcción de un individuo autónomo. El individuo es tanto más autónomo cuanto más plenamente socializado mas referido directamente a valores asumidos como suyos está, lo que permite construir un juicio autónomo y racional. En efecto, en las sociedades modernas los valores últimos son los de la razón. De este modo esta sociología puede revestir un aspecto "encantado" puesto que no establece contradicción entre la libertad y la socialización. El protestante de Weber, el individuo "de corte " de elias, o el hombre "inner directed" de Riesman, son igualmente figuras heroicas de este individualismo moderno. Evidentemente, estos sociólogos no son ingenuos, ven también las guerras, las masacres, los totalitarismos amenazantes o realizados, pero postulan un horizonte de razón posible, de la misma manera que Manx veía en el movimiento obrero un sujeto colectivo mas allá de la alineación cotidiana. 
¿Ocaso de la idea de sociedad?

\section{EL OCASO DE LA IDEA DE SOCIEDAD}

Esta representación de la idea de sociedad se deshizo durante los últimos cuarenta años por razones teóricas e históricas. Esta descomposición es extremadamente compleja, y, por motivos de claridad, distinguiremos dos etapas en el pensamiento social contemporáneo.

\section{La sociología crítica}

Por convención definiré la sociología crâica como aquella que conserva la idea clásica pero que la invierte enteramente desde el punto de vista ideológico y normativo. Es una suerte de contramodelo paralelo a la idea de sociedad que, manteniendo los principios de razonamiento, invierte los principios ideológicos y morales. Sin duda esta sociología crítica siempre ha existido, pero es a fines de los años sesenta cuando se impuso por una decena de años.

a) La moclernidad no se identifica a la razón sino a la dominación occidental. El progreso es desnaturalizado por el capitalismo y el imperialismo que frenan el desarrollo. Ahi donde el cambio podria aparecer como endógeno y "natural", la ruptura revolucionaria debe asumirlo y realizarlo.

b) La idea del sistema social no es rechazada. Por el contrario, se refuerza por la identificación de la sociedad y de un principio de dominación en forma de un "funcionalismo invertido" (Bourricaud, 1987). La cultura deviene la ideología dominante, la división del trabajo una forma de explotación, el consumo una modalidad de la alienación y la integración social se reduce al control social. Toda esta inversión aparece claramente en la evolución de las teorías de la desviación y de la educación, por ejemplo.

c) No se cuestiona directamente la identificación de la sociedad con el Estado, contrario, la democracia es percibida como "formal", como una modalidad de la dominación. La integración progresiva de los actores sociales es reemplazada por la construcción de un horizonte revolucionario.

d) La idea de conflictos de clases regulados es sustiturda por la de ruptura revolucionaria. Pero en una sociedad considerada como un sistema general de dominación y de alineación, los movimientos sociales no pueden tener ya el primer rol. Ellos deben entonces ser guiados o reemplazados por las vanguardias. Es la retoma de esta tradición intelectual, leninista mas que marxista, la que puede definir a la sociologia crítica como "gauchista" (izquierdista), bastante diferente de lo que se llamaba izquierda en el modelo clásico. 


\section{La crítica de la idea de sociedad}

Paradojalmente puede considerarse a la sociologia crica como el canto del cisne de la sociología clásica. Esta muere invirtiéndose en un modelo que es su negativo. Y la agonia fue breve. Desde los años setenta y ochenta, loda una serie de mutaciones sociales, a nivel mundial, hacen poco crebles tanto la idea clásica de sociedad como su versión crítica. Citemos sin mayor orden: el fin del mundo comunista; el fracaso de los gobiernos emergentes de la descolonización; la extensión considerable del mercado internacional, las contrarevoluciones populares e integristas en el mundo musulman; la manifestación de minorías étnicas en el seno de las sociedades mas desarrolladas; la pérdida de confianza en la ciencia con la ecologia; la formación de nuevos movimientos sociales y, mas ampliamente, la toma de conciencia de las catástofes de la modernidad que, de acuerdo con la tesis de la Escuela de Francfort, instauró mas bien el reino de la racionalidad instrumental y no el de la razón. Todos estos cambios no tienen siempre efectos directos en las teorías sociologicas contemporáneas, pero explican por qué estas teorías, antiguas y redescubiertas o mas recientes, desestabilizan la idea de sociedad, y pueden instalar el reino de una sociología sin sociedad.

a) El par formado por la oposición entre la tradición y la modernidad conserva un cierto valor, pero no está ya en situación de definir la naturaleza de las sociedades en una suerte de jerarquía de modernización.

En efecto , en el seno del mundo capitalista han aparecido varios tipos de modernización (Barrington Moore) en los cuáles el desarrollo económico, la democracia y el desarrollo cultural no están necesariamente asociados. Dicho de otra manera, ya no es posible definir una sociedad como globalmente moderna o tradicional. Los Estados Unidos conocen movimientos religiosos en un alto grado "arcaicos" mientras que sociedades menos ricas pueden ser culturalmente mas "modernas". La decadencia del evolucionismo latente de la sociología está también ligada a la emergencia de movimientos sociales críticos de la modernidad y de la ciencia, sin ser por ello movimientos conservadores. La confianza en la "fuerzas productivas" se quebró. El horizonte evolucionista salido del pensamiento del siglo XIX no permite ya pensar en el cambio.

b) El cambio térico más espectacular obedece sin duda a la declinación del pensamiento funcionalista. A la concepción holista de un sistema que engloba y determina las conductas de los individuos a partir de funciones elementales, le sucede un "sistema emergente" concebido como un equilibrio mas o menos estable que es el resultado de la suma de las acciones y de las elecciones de los individuos. La antigua metáfora organicista es reemplazada por la del mercado. El área de investigación que ilustra mejor este cambio es el de la sociología de las organizaciones. En un primer momento las organizaciones son definidas como 


\section{¿Ocaso de la idea de sociedad?}

conjuntos funcionales, mas tarde van siendo definidas poco a poco como los equilibrios que resultan de las múltiples estrategias de los actores que las componen (March y Simon, Crozier y Friedberg). El tema de las organizaciones cerradas es reemplazado por el de las organizaciones abiertas a las fronteras inciertas con su medio ambiente.

c) No parece ya posible identificar con la misma certeza de principios de siglo la idea de sociedad con la de Estado-Nación.

La unidad de una economía, de una gran cultura y de un Estado parece deshacerse. En Francia, donde esta creencia ha sido muy fuerte, esta crisis es profundamente sentida y puede explicar, en parte, el poder de la extrema derecha. Primero, las economias nacionales aparecen cada vez más como ficciones en razón de su integración internacional. El "personaje" nacional pierde el control de su acción y de su medio ambiente económico. Luego, la idea de cultura nacional, a la vez especffica y universal, aparece amenazada de dos maneras. Por un lado, está confrontada a la cultura internacional de los medios de comunicación de masas y de los modos de consumo que proponen: la televisión, la música pop y la publicidad deben cada vez menos a las especificidades culturales nacionales. Por otro lado, se obsenva en las sociedades mas modernas reivindicaciones identitarias étnicas, culturales y religiosas en donde la idea de mosaico sustituye la de nación homogénea. De hecho, los individuos viven en varias culturas que tienen áreas de extensión diferentes. En fin, la imagen de la vida política se transforma con las mutaciones de la representación política, la ampliación del campo de intervención de las politicas más que de la política, y la proliferación de los lugares de poder.

d) La idea de sociedad estaba totalmente asociada a la de sociedad industrial. Es en las categorías de la sociedad industrial donde se definía la modernidad, las clases sociales, los movimientos sociales. Ahora bien, salimos de la sociedad industrial sin conocer con el mismo rigor las categorias de la sociedad posindustrial. La tecnología se mezcla al capitalismo, las clases medias y los empleados del terciario constituyen la mayor parte de la sociedad. Una cierta dualización se instaura en muchas sociedades "modernas". Los grandes aparatos de cultura de masas, de educación y salud por ejemplo, acrecientan su control sobre la vida privada y la definición de necesidades. Se observa en todas partes una decadencia del movimiento obrero en tanto movimiento social "total" capaz de defender intereses especfficos y de promover un contra proyecto político y social. La idea según la cual una sociedad puede ser estructurada por un conflicto central y regulado, deja ya de imponerse. Los nuevos movimientos sociales no ocupan el lugar dejado vacante por el movimiento obrero. Anotemos que desde una veintena de años cesan de emprenderse tentativas de construcción sistemáticas del modelo de sociedad posindustrial (Bell 1976, Touraine, 1969) en beneficio de visiones mas parceladas. (Ball 1978, Luhman 1982). 


\section{LA SEPARACIÓN DEL ACTOR Y DEL SISTEMA}

\section{Subjetividad y Objetividad}

La declinación de la idea clásica de sociedad arrastra necesariamente a las teorías sociológicas hacia la confirmación de la distancia, sino de la ruptura, entre el actor y el sistema, entre la subjetividad de los individuos y la objetividad de los "hechos sociales". Esta separación es el trasfondo común de las teorías sociológicas contemporáneas, tanto si se trata de corrientes nuevas o de escuelas o autores redescubiertos. Esta separación se manifiesta bajo formas múltiples. Al riesgo de caricaturizar, pueden evocarse algunas, como la oposición entre holismo e individualismo, entre "micro" y "macro", entre acción e interacción. Como lo formula Habermas con nitidez, se crea una distancia creciente entre el mundo de la vida y la subjetividad y el mundo del sistema dominado por la racionalidad instrumental.

De la misma manera, puede insistirse sobre el lugar, hoy central de las filosofías políticas que constatan la diversidad y la incompatibilidad de los valores (Rawis 1987, Walser 1990, Boltansky y Thevenot 1987). La justicia no es ya percibida como una norma sino como una combinatoria de principios diferentes y como una actividad práctica.

A fin de cuentas, la sociología contemporánea es una sociología de los actores que lleva, como mayor o menor claridad, a ahorrarse la idea de sociedad.

\section{Tres líneas de razonamiento}

No se trata de trazar el panorama general de la sociología actual en el marco de este breve texto. Pero puede decirse, en grueso, que tres grandes sensibilidades se reparten el campo intelectual.

a) La primera conserva el razonamiento clásico asociándole un punto de vista crítico. Bourdieu encarna principalmente esta orientación, a la vez clásica y desencantada, colocándose bajo el triple patronazgo de Max, Weber y Durkheim. La noción de habitus tiene por función rechazar la dualidad de la subjetividad y la objetividad, incluso si no escapa, me parece, a un objetivismo que obedece a su propio desencantamiento.

b) La segunda línea de razonamiento es de inspiración "fenomenológica". Ella se preocupa por la manera como los actores construyen la realidad y los intercambios sociales. Aqui puede establecerse una gradación hacia una ruptura cada vez mas radical con la idea de sociedad. Mientras que Berger y Luckman están aún próximos del modelo clásico por muchos aspectos, el interaccionismo 


\section{¿Ocaso de la idea de sociedad?}

simbólico, particularmente la obra de Goffman, se aleja mucho mas nítidamente. La idea de sociedad aparece aquí como una hipótesis inútil, de la misma manera que la idea del yo es reemplazada por la de cara en donde el sujeto es un "empresario" que busca promover sus intereses y mantener la reacción social. Sin duda se puede intrepretar Goffman de un manera menos cínica, pero yo me inclino en ver ahí su principal fuerza y su capacidad de describir sin romanticismo los modos de vida de las clases medias utilitaristas. Más radical aún es la ruptura introducida por la etnometodología que concibe la sociedad como una realización práctica donde la misma idea de sociedad está definida como una manera, entre otras posibles, de dar cuenta de la realidad. La vida social se reduce a una interacción de lenguaje. No se trata de criticar estas teorías, sino de subrayar el hecho de que no recurren ya a la idea de sociedad, la de vida social es suficiente. Estas socioloías devienen solamente "micro" y reducen la vida social a los intercambios individuales. Tampoco descansan en teorías previas de la estratificación, del poder, del cambio...de todos los objetos esenciales de la sociología clásica. El riesgo que corren estas orientaciones es construir conocimientos "insignificantes" del punto de vista de lo que está en juego en la vida social.

c) El tercer eje de razonamiento es de inspiración racionalista y utilitarista. El actor inscribe sus opciones en una racionalidad limitada en relación a lo que él sabe de su situación, de sus recursos y de los otros actores. El conjunto del contexto social y cultural es tratado como un stock de recursos que los individuos movilizan para alcanzar fines definidos en términos de utilidad. Las teorías de la movilización colectiva en términos de "rational choice" y las teorías de la organización en términos de zonas de incertidumbre participan de esta representación. Esta visión conduce a una crítica severa del "holismo", crítica que no es solamente metodológica, sino que involucra la idea de sociedad. Los fenómenos colectivos aparecen como los efectos emergentes, incluso perversos (Blau, Jenks, Boudon), la prioridad atribuida a la racionalidad conduce al estudio de la argumentación en una perspeciva cognitivista en la cual los contextos sociales de la comunicación ya no son necesarios (Boudon.)

No se trata de impugnar estas perspectivas ni de ignorar su saber adquirido. Éstas han transformado profundamente nuestra manera de concebir la acción social, pero quisiera subrayar que no se apoyan ya en la idea clásica de sociedad, y que en eso, participan de una verdadera mutación de la sociología definida como una filosofia social de la modernidad. Sin embargo, no se puede ignorar el riesgo de una evolución radical hacia la disociación creciente entre los problemas sociales y las mutaciones históricas, por una parte, y las ambiciones de las ciencias sociales, por la otra. 


\section{REVISTA DE SOCIOLOGLA}

\section{DEL ROL A LA EXPERIENCIA}

En el fondo, la sociología contemporánea no asume ya "ingenuamente" la noción del rol, no cree en la unidad de la personalidad y la sociedad, además, sabe que los actores producen el mundo en el que viven. Al mismo tiempo, con la declinación de la idea clásica de sociedad, la unidad de esta sociedad se fractura porque no aparece constituida ya por un principio fundamental en torno a los valores y a un conflicto central. Es necesario sacar las consecuencias de ello afirmando que la unidad del rol es reemplazada por la diversidad de la experiencia. Dicho de otra manera, cada uno de nosotros es un actor en la medida en que debemos administrar la diversidad de las lógicas de acción que se encuentran en su experiencia (Dubet 1994).

\section{Las lógicas de la experiencia social}

Pueden distinguirse tres lógicas fundamentales de la acción que se conjugan en la experiencia social de cada uno.

a) La integración define la identidad de manera clásica como una interiorización cultural. Esta identidad, a menudo, es vivida como un ser, como una "naturaleza". En este registro las teorías del tipo durkheimiano, y en una amplia medida la del habitus son perfectamente aceptables. Conviene, sin embargo, agregar que esta lógica de la acción es una actividad en la medida que cada uno trabaja para "producir" esta identidad que opone "nosotros" a "ellos", las raíces al cambio, la pertenencia a la exclusión. El hecho de que esta lógica se rutinice a menudo no impide recordar que uno de los objetos más antiguos de la sociología es el análisis de las conductas sociales de crisis, es decir, acciones de defensa de una identidad y de una pertenencia amenazadas.

b) La acción estratégica construye una lógica en la cual los individuos se definen por sus recursos y su racionalidad. La situación social es, entonces, definida en términos de competencia, cada uno es el rival de todos, y los términos de pertenencia y diferencia son sustituidos por los de alianzas y rivalidades. Esta lógica de "mercado" no es sólo válida para el dominio económico, vale también para todos los "campos posibles", cultural, amoroso, escolar...

c) La subjetivación. Más allá de estar compartidos entre la integración y el mercado, define a los individuos como sujetos capaces de reflexión y de crítica. Esta distancia de sí, que define al sujeto, descansa necesariamente en una definición cultural del sujeto como representación de la autenticidad y de la creatividad humana. Pero el individuo mismo no es un sujeto, sólo lo deviene en la experiencia de la separación de sí que le permite determinar su vida y percibirse como su autor. 


\section{¿Ocaso de la idea de sociedad?}

Durante bastante tiempo esta representación del sujeto fue situada "fuera del mundo" (Dumont, 1983). Después entró poco a poco en el mundo, bajo la forma de la razón, del trabajo, de la atenticidad... Es esta referencia al sujeto lo que hace que los actores no se reduzcan ni a sus intereses ni a sus pertenencias. Cada objeto social tiene que ver con estos tres tipos de lógicas. La religión, por ejemplo, es a la vez una moral colectiva que garantiza la integración de un grupo (Durkheim), es un recurso ideológico que permite establecer las estrategias de dominación (Marx), es también una ética no social que garantiza una distancia en relación al mundo y una distancia de sí a sí (Weber). De hecho, es todo a la vez, es a la vez el orden, el diablo y el buen Dios. Pasa lo mismo en experiencias mas banales como el amor (Martucelli, 1994) donde el discurso se organiza según tres registros esenciales; la institución conyugal, el sujeto romántico y la estrategias de seducción. Es a la vez, la familia, Tristán y Don Juan.

\section{Lógicas separadas.}

Se debe hablar de experencia social en la medida que estas lógicas de la acción se separan de manera creciente, donde su unidad no está dada a los actores, e imponen a los individuos una exigencia de recomposición. Tomemos algunos ejemplos: los movimientos sociales, la escuela, las conductas desviadas.

a) La imagen del movimiento obrero se impuso por bastante tiempo como un movimiento social "total" que tenfa como atributos la capacidad de integrar sólidamente la defensa de una comunidad, la lucha por intereses especfficos y la identificación con un proyecto socledad, una utopla. Las tensiones entre estas lógicas no parecian insuperables, en todo caso podían ser superadas en lanto que obviamenie se imaginase un principio de coninuidad entre la fusión dentro de la comunidad, de la diversidad de intereses y el proyecio colectwo. El movimieno obrero, al igual que la scciedad, fue un personaje. Desde muchos años se observa una disociacion de estas lógicas de acoín: la comunidad se repliega o se disuelve en la sociedad de masas, la racionalidad corporativa no excede los intereses específicos y el contra proyecto social se escinde de la clase obrera deviniendo únicamente crítica intectual y moral. El movimiento obrero cede su lugar a las políticas como una de las tantas modalidades de una experiencia que no tiene ya unidad (Touraine, Wieviorka, Dubet). $Y$ en cuanto a los nuevos movimientos sociales, éstos se construyen sobre la base de esta disociación.

b) Un razonamiento paraielo puade ser cusarollado a propósito do la escuela. Lo que se llama apresuradamente, escuela republicana en Francia, se ca. racteriza por la sólida integración de las funciones esanciales de todo sistema escolar. La función de distribución de las calificaciones escolares, la función de so- 


\section{REVISTADE SOCIOLOGIA}

cialización al interior de una forma escolar y la función de educación en un modelo cultural progresista, eran afirmadas e integradas. La condición de esta integración obedecia ampliamente al rol a la vez dominante de la segregación escolar que conducía los individuos hacia escolaridades diferentes en función de su nacimiento, y al rechazo a dejar un lugar a la infancia y la juventud en el seno de la vida escolar. La escuela funcionaba como una institución. La masificación escolar, por una parte, y el desarrollo de la autonomía de la juventud, por la otra, desestabilizaron esta representación de la escuela y de la socialización (Dubet 1996, Martucelli). Con la masificación la escuela se contruye como un "mercado" de las calificaciones y es la escuela misma quien organiza los modos de distribución de las competencias. Los alumnos se identifican con una cultura juvenil de masas que funda ampliamente sus identidades y la cultura escolar, con lo que tiene de crítica y de universalidad, se separa a la vez de la integración juvenil y de los intereses sociales construidos en la escuela. De esta manera, la socialización se efectúa en la experiencia de los alumnos, es decir, en sus capacidades o incapacidades para combinar estas lógicas. Alumnos y profesores deben combinar las significaciones cuya integración no está ya dada por la institución.

c) Podría describirse la misma evolución para la familia que se define más como un "arreglo" (de Singly 1987), que como una institución. En el caso de las conductas marginales urbanas (Dubet), he intentado mostrar que la experiencia de la galére * se construia como una combinación inestable entre las tres lógicas de la experiencia: la anomia ligada a la descomposición de las comunidades populares, la exclusión económica y escolar intensamente mas sentida por los jóvenes en la medida que adhieren a los valores de la cultura de masas, y la "rabia" surgida de la declinación de la conciencia de clase obrera capaz de transformar un sentimiento de dominación en conflicto social. Para responder a las exigencias de estas tres lógicas, los jóvenes crean bandas territoriales y étnicas, se comprometen en un conformismo desviado, y son llevados por la cólera que se manifiesta en los motines de los sectores periféricos.

\section{El trabajo del actor}

La disociación de las lógicas de la acción conduce hacia la afirmación de dos principios de análisis.

a) El sujeto es un trabajo. En las sociedades donde las lógicas de la acción aparecen cada vez mas autónomas, los actores colectivos y los individuos deben

\footnotetext{
Expresión usada para referirse a la experiencia de vida de los jóvenes marginales de los ochenta, su oposición a las "bandas" de los 50 y 60 y a la rebeldía política y cultural de los 60 y 70 (Dubet ,1987. Nota de los traductores)
} 


\section{¿Ocaso de la idea de sociedad?}

necesariamente construir sus experiencias. Deben combinar los registros de la acción, construyendo el sentido y la coherencia. Es esta actividad la que construye el sujeto social en la medida que implica una distancia de sí y una reflexividad. Pero esta definición no es suficiente en la medida que el trabajo del actor moviliza también la referencia a una definición histórica del sujeto y la conciencia de los obstáculos a su realización. La conciencia de sí exige la referencia cultural a la creatividad humana, a la vida buena, a la autenticidad... que son otras tantas definiciones de la naturaleza humana. Sin embargo, los actores se experiencian como sujetos sólo en la prueba que los separa del sujeto, particularmente en la dominación social. El sujeto individual y social se construye en una relación al sujeto cultural e histórico que es como el Dios escondido.

b) Las lógicas de la acción son objetivas. Esta representación del actor y la acción social está centrada en la subjetividad de los actores. Pero no debe llevar a una visión puramente subjetiva de la experiencia puesto que cada lógica de la acción es objetiva y, como tal no pertenece a los actores. Esta afirmación no afecta la heterogeneidad de los principios de la acción porque cada lógica depende de un modo de determinación particular. La lógica de integración se inscribe en los procesos objetivos de socialización de la misma manera que si cada uno tiene un habla individual solo puede hablar en una lengua común y objetiva que existe sin él y le impone los valores y las reglas coactivas de expresión. La lógica estratégica remite a una determinación que es la del jugador. El espacio del juego, las reglas del juego y los recursos de los jugadores, están objetivamente distribuidos y algunos tienen mas márgenes de iniciativa que otros. La metáfora de la competencia y el mercado no es la de la libertad sino la de la racionalidad mas o menos amplia. En fin, la definición del sujeto es, también, objetiva porque es una creación cultural. Más aún, las relaciones sociales definen la distancia a esta figura del sujeto en una casualidad de tipo "dialéctico". De esta manera se comprende mejor cómo se puede observar un aumento de la distancia subjetiva del actor y del sistema, manteniendo la idea de una determinación global de los registros de la acción. Ni el actor ni la sociedad descansan ya en un principio central que funda su unidad respectiva. Dicho de otra manera, si la experiencia social es una actividad y una creación de los actores, sólo se construye a partir de datos objetivos y es por este desvío que no invita a no abandonar la idea de sociedad.

\section{CAMPO SOCIAL Y SOCIEDAD}

\section{Campo Social}

La primera manera de defender la idea de sociedad es recurrir a una imagen débil, la de una yuxtaposición de campos sociales. $Y$ consiste en recordar que ca- 


\section{REVISTADE SOCIOLOGIA}

da lógica de la acción se sitúa al interior de un espacio global, formado por un subsistema estructurado que tiene sus propias "leyes", más allá de los diferentes dominios de actividad, desde la integración él está definido por un conjunto de roles, de status, de normas y de modelos morales. Desde este punto de vista no se debe abandonar ni las nociones de estratificación ni la de organización. La dificultad actual viene de que las diferenciaciones funcionales se han multiplicado, de acuerdo con la narración de la modernidad, y de que los campus de integración son extraordinariamente numerosos, ya que los individuos participan en una multiplicidad de ellos. Esto explica, por lo demás, la fuerza de las descripciones de Goffman, en la medida que muestra bien cómo cada uno guarda para sí una parte del decorado escondido donde los juegos de lo público y lo privado, de la exposición y el secreto, han devenido numerosos y sofisticados. Se han formado otros campos que son, a la vez, más masivos y más efímeros, como los públicos constituidos por los medios de comunicación de masas que pueden crear sentimientos e identidades. Se observa un vaivén creciente de lo público y de lo privado. Dentro del registro de la iniegración, la búsqueda de creencias en las normas culturales comunes capaces de superar la diversidad de los campos, conduce, en definitiva, a identificar la sociedad a la nación.

De la misma manera, puede considerarse que los diversos espacios estratégicos están también fuertemente estructurados por reglas explicitas o latentes, y por una distribución objetiva de los recursos. Puede trazarse entonces todo un conjunto de espacios estratégicos estructurados como otros tantos "mercados", y mostrar cómo los actores transforman las reglas jugando (Reynaud 1989). ¿Cuáles son las reglas generales y cuáles las particulares? ¿Cómo se transforman?. Esta visión lleva necesariamente a atribuir la mayor importancia a la acción política como principio de regulación. Desde este punto de vista, la unidad de la sociedad estaría conferida por la actividad política y, para decirlo claramente, por el Estado.

En realidad, la dificultad de una teoría de los campos conduce necesariamente a una visión parcelada de la sociedad, de la cual, en una cienta medida, las especializaciones sociológicas son el reflejo. La sola manera de mantener una unidad del conjunto sería postular una superposición de campos y de separaciones en niveles diferentes según un principio de dominación único, como los propone Bourdieu. Pero esta representación parece difícil defender en una sociedad que es a la vez una sociedad de masas, compleja y, en la cual, los individuos están en la encrucijada de campos múltiples y heterogéneos. La concepción de la sociedad en términos de campos ofrece una imagen de la vida social pero no reemplaza realmente la idea de sociedad. Esta conduce hoy a definir "concretamente" las sociedades como el encuentro de una nación y una economía mundial, como una manera en la cual una política nacional combina estas dos dimensiones. $Y$ reconozcamos que esta imagen tiene fuerza y actualidad. Después de todo, es la de Europa de hoy como fue la de la Europa mercantil del siglo XVI. 


\section{La Sociedad}

La idea de sociedad es más fuerte que la de campos de la vida social porque implica que la combinación de nación y mercado se estabiliza a partir de principios generales a la vez culturales y sociales, como fue la alianza entre modernidad y capitalismo, es decir, la apropiación de la modernidad por el capitalismo y la impugnación del capitalismo en nombre de la modernidad.

¿Se puede retomar este tipo de razonamiento hoy?. Ahí está toda la problemática de la construcción de la idea de sociedad. Es conveniente, entonces, volver a la definición de un modo de dominación social y a la representación histórica del sujeto. En lo que se refiere a la definición del sujeto, ésta parece más cómoda puesto que se concebirá fácilmente que se trata del individuo capaz justamente de construir su experiencia. El sujeto se define como el llamado a una autenticidad entre el individualismo de mercado y el individualismo moral (Taylor, 1989). Parece mas complejo definir el modo de dominación social de una sociedad post industrial, en la medida en que esta dominación no tiene centro y no está ciertamente encarnada por un grupo relativamente unificado como pudo serlo la burguesía. El tema del mercado por sí solo no es suficiente porque una clase dirigente se define por sus proyectos, y la dificultad es tanto mayor hoy día cuanto estos actores y sus proyectos desbordan ampliamente el marco nacional. ¿Se trata de la tecnocracia?, ¿Se trata de los que se identifican con la racionalización creciente del mundo?. La pregunta queda abierta, pero no puede ahorrarse una reflexión nueva acerca de la sociedad posindustrial. En todo caso, ya no se puede identificar la idea de sociedad a la de sistema sino a la de un modo de combinación de muchos sistemas autónomos.

Si no se puede ya recurrir a la idea clásica de sociedad, no creo que hayamos entrado en el puro desorden o en el encuentro puramente aleatorio de muchos campos. En este sentido no se debe ser posmodernos, sino más bien neomodernos. Si la sociología abandona la idea de sociedad está amenazada de devenir insignificante, de reducirse al estudio de las interacciones cuando la antropología y la historia hablarán mejor de la nación, y cuando los economistas y los cognitivistas hablarán mejor de la racionalidad instrumental. Queda, entonces, por construir un horizonte de filosofía social si no se quiere que la sociología haya sido sólo la aventura intelectual de la modernidad de las sociedades industriales nacionales. 


\section{REVISTA DE SOCIOLOGIA}

\section{BIBLIOGRAFIA}

Bell, D.,

Bell, D.,

Berger, P. y

Luckmann, T.

Boltanski, L.

Thévenot, L..

Boltanski, L. y

Thévenot, $L$.

Bourdon, R.

Bourdieu, P.

Bourricaud,F.

Crozier, M. y

Friedberg, $\mathrm{E}$.

Davis, $\mathrm{K}$.

Dubet, F.

Dubet, Fy

Martucelli, D.

Dumont. L.

Garfinkel, H

Gellner, E.

Goffman, E.

Luhmann, N.
Vers la societé post-industrielle, Paris, R. Laffont, 1976.

Les Contradictions culturelles du capitalisme, Paris, PUF, 1978

La constructión sociale de la réalité, Paris, Méridien-Klincksieck, 1986.

Les économies de la grandeur, Paris, PUF, 1987.

De la justification. Les économies de la grandeur, Paris, Gallimard, 1991.

La logique du social, Paris, Hachette, 1979. L'idéologie, Paris, Favard, 1986.

Le sens pratique, Paris, Ed de Minuit, 1980.

Contre le sociologisme: une critique et des propositions, Revue Française de Sociologie, XXVIII, 1, 1987, p. 43-65.

L'acteur et le système, Paris, Ed du Scuil, 1977.

Le mythe de l'analyse fonctionnelle, in Mendras, $\mathrm{H}$., Elèments de sociologie, Textes, Paris, A. Colin, 1968.

La galère. Jeunes en survie, Paris, Fayard, 1987. Sociologie de l'expérience, Paris, Ed du Seuil, 1994.

A l'école, Paris, Ed du Seuil, 1996. (A paraître).

Essais sur l'individualisme, Paris, Ed du Seuil, 1983.

Studies in Ethnomethodologies, New York, Prentice hall, 1967.

Nations et Nationalismes, Paris, Payot, 1989.

Les cadres de l'expèrience, Paris, Ed de Minuit, 1991.

The Differentiation of Society, New York, Columbia University Press, 1982. 
¿Ocaso de la idea de sociedad?

March, J.G y

Simon, H. A Les organisations, Paris Dunod, 1991.

Martucelli, D. Décalages, Paris, PUF, 1994.

Merton, R.K Eléments de théorie et méthode sociologique, Paris Plon, 1965.

Moore, B. Les origines sociales de la dictadure et de la démocratie, Paris, Maspero, 1979.

Nisbet, R. A. $\quad$ La tradition sociologique, Paris, PUF, 1984.

Rawls.J. Théorie de la justice, Paris, Ed du Seuil, 1987.

Reynaud, J.D. Les règles du jeu. L'action collective et la règulation sociale, Paris A Colin, 1989.

Singly, F.de Fortune et infortune de la femme mariée, Paris, PUF, 1987.

Touraine, A. La societé post-industrielle, Paris, Denoël, 1969.

Touraine, A.

Wieviorka, M. y

Dubet, $F$.

Le mouvement ouvrier, Paris, Fayard, 1984.

Taylor, C. Sources of the Self, Cambridge University Press, 1989.

Walzer, M. Critique et sens commun. Essai sur la critique sociale et son interprétation, Paris, la Découverte, 1990. 